\title{
EasyChair Preprint
}

\section{Energy Consumption Improvement based on Distance Adaptive Modulation in Optical Elastic Network}

Sabi Yari Moïse Bandiri, Rafael M.S. Braga, Tales C. Pimenta and Danilo H. Spadoti 


\title{
Energy Consumption Improvement based on Distance Adaptive Modulation in Optical Elastic Network
}

\author{
Sabi Y.M. BANDIRI ${ }^{1,2}$, Rafael M.S. BRAGA ${ }^{1}$, Tales C. PIMENTA ${ }^{1}$ and Danilo H. SPADOTI ${ }^{1}$
}

\begin{abstract}
In this paper, a novel algorithm based on distance adaptive modulation routing spectrum assignment is presented. The shortest energy consumption Routing Spectrum Assignment (SEC-RSA) algorithm was developed, based on Distance Adaptive Modulation (DAM). According to the distance between source and destination, for a determinate lightpath, the modulation pattern is modified. The energy consumption is computed for the $k$-shortest path and, then, the one with lowest energy consumption is adopted. The algorithm can dynamically commute to another modulation as the connections requests or the numbers of lightpath between source and destination increases. The results show that the energy consumption decreases when SEC-RSA algorithm is used in comparison with traditional ones. Energy consumption decreases as the order of modulation increases. The results also figure out that energy consumption decrease when block connections are considered.
\end{abstract}

keywords: Energy Consumption, Distance Adaptive Modulations, Switching dynamically

\section{INTRODUCTION}

Growth in the use of all types of Information and Communication Technologies (ICT) led to the rapid increment of energy consumption across the world. Energy consumption is becoming a key environmental, social and political issue [1]. Many countries and institutions are investing and developing techniques and algorithms with capacity to reduce the energy consumption in ICTs. As example, European Union Organization has set-up a goal to reduce $20 \%$ of energy costs until 2020 and the Japanese government work out the same line of sight [1], [2]. To this end, several researches have being performed numerous techniques and algorithms aiming to reduce energy consumption [1]-[3].

Wavelength division multiplexing (WDM) networks appears as a auspicious way to improve optical network performance. However, this technology introduces some critical impairments, such as: density of channel spacing, different nonlinear effects (Raman and Brillouin scattering, four-wave mixing (FWM), cross-phase modulation (XPM), self-phase modulation (SPM)), among others. By the way, elastic optical networks (EON), which uses Orthogonal Frequency Division Multiplexing (OFDM) and an advanced modulation techniques, have been employed as a potential candidate to overcome the WDM limitations. This scheme has been projected as a technique to allocate bandwidth to optical paths (referred as lightpath), based on their required

\footnotetext{
${ }^{1}$ Institute of Electrical Systems and Energy,Federal University of Itajubá, Av. B P S, 1303 - Pinheirinho, MG, 37500-903

${ }^{2}$ Corresponding Author: bandiri@unifei.edu.br
}

transmission rates [4]. When a new connection is solicited, an EON considers the best implementation of the request, in terms of modulation format, Forward Error Correction (FEC), and spectrum, yielding the lowest-cost solution. This decision is based on adaptive behavior, that adjusts itself based on actual link conditions and control plane knowledge of impairments in the network, while still taking into account margins for network aging [5].

Elastic optical networks uses the routing and spectrum assignment (RMSA or simply RSA) technique [6]. Designing efficient RSA algorithms has gained an attractive attention and considerable methods have been introduced to establish a connection between a source and destination, which is called as unicast routing [6], [7].

A distance adaptive routed spectrum assignment (DARSA) algorithm considering various types of traffic with different quality of-service (QoS) requirements was proposed in [6]. The traversed distance was the only quality of transmission factor taken into account. In [7], a new impairment-aware RSA algorithm in flexible OFDM-based networks was recently studied, but only BPSK was used as modulation. The question of how to improve the spectrum utilization under the constraint of physical impairments remains to be the important open issue in flexible optical networks.

Therefore, as a main contribution of this paper, a novel strategy, based on Distance Adaptive Modulation (DAM), is proposed, whereby the energy consumption can be reduced. The proposed algorithm, based on distance awareness, dynamically allocates the appropriate modulation for every lightpath. The number of lightpath, $k$, between source and destination is not fixed value, instead it dynamically vary according to the connection request. This proposed shortest energy consumption Routing Spectrum Assignment SECRSA Algorithm ensures quality of-service (QoS) and quality of-transmission (QoT) criteria.

The remaining of the paper is organized as follows. The model of energy consumption over optical network is described in section 2. In section 3, a novel algorithm SECRSA is introduced and the results are discussed in section 4. Finally, the paper is concluded in section 5 .

\section{BUILDING BLOCKS OF OFDM SYSTEMS FOR OPTICAL LIGHTPATHS AND POWER MODEL}

In this proposal study, a generic building block diagram of an OFDM system, illustrated in Fig.1 is considered. The block diagram represents the set of components, such 
as: transmitter, optical fiber spans with optical amplifiers, bandwidth variable optical cross-connects (BV-OXCs), and receiver. This diagram block is not stationary, instead it varies according to systems specifications, bit rate, and distance between nodes.

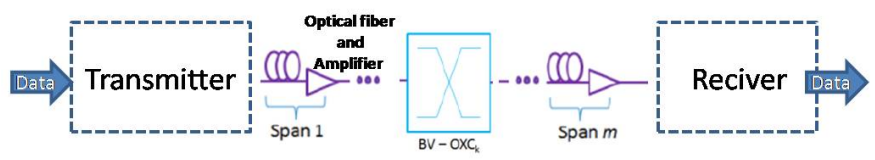

Fig. 1. Block diagram of end-to-end lightpath OFDM-based transmission system

For a determinate lightpath OFDM-based, the total energy consumption, $P_{\text {ptical }}$, can be computed as following [8]:

$$
\begin{array}{r}
P_{\text {Optical }_{i}}=P_{T X_{i}}+P_{R X_{i}}+\sum_{q=1}^{Q} P_{E D F A(q))_{i}}+ \\
\sum_{r=1}^{R} P_{B V-O X C(r)_{i}}+\sum_{r=1}^{R} P_{\text {Control }(r)_{i}}
\end{array}
$$

In (1), $P_{T X_{i}}$ and $P_{R X_{i}}$ are the power consumption of the transmitter and the receiver, respectively. $P_{E D F A(q)_{i}}$ is the power consumption of the qth Erbium Doped Fiber Amplifiers (EDFAs) in the optical route (booster, in-line, in the BV-OXCs and at the receiver), $P_{B V-O X C(r)_{i}}$ is the power consumption of each $r$ th BV-OXCs. Finally, the control plan power consumption is defined by $P_{\text {Control }(r)}$.

The way to compute the power consumption in each block, step by step, (transmitter, receiver, amplifiers, BX-OXC) of Fig.1 can be found detailed in the reference [8]. In this work, Binary Phase Shift Keying (BPSK), QuadraturePSK (QPSK), 8-PSK and M-ary Quadrature Amplitude Modulation (M-QAM), where $\mathrm{M}=16,32$ or 64 symbols were used. Each one of these modulations provides different type of spectral efficiency, expressed in bit/s/Hz. A single sub-channel with $12.5 \mathrm{GHz}$ of bandwidth can be 12.5, 25.0, 37.5, 50.0, 62.5 and 75.0 Gbps for BPSK, QPSK, 8-QAM, 16-QAM, 32-QAM and 64-QAM, respectively, depending on the optical channel state condition.

The Maximum Distance Transmission (MDT) reached per modulation can be computed through the following equation in which $12,5 \mathrm{GHz}$ as a sub-channel bandwidth was considered [8]:

$$
D_{\max }=\frac{1}{2}\left[-804,3 \times \ln \left(B_{R}\right)+5584,6\right]
$$

In (2), $D_{\max }$ denotes the maximum transmission reached in $K m$, and $B_{R}$ denotes the bit rate in Gbps. The above equation satisfies the impairments issues mentioned in WDM technologies and therefore, the QoS and QoT.

\section{SEC-RSA ALGORITHM}

Distance adaptive modulation concept presented in [4] was used, aiming to reduce energy consumption in EONs. The main idea of SEC-RSA is as following: the constellation scheme of a modulation varies as function of the lightpath distance among source and destination. If a lightpath distance is longer than the transmission distance reached by a determinate modulation, the number of modulated bit per symbol transmitted is dynamically modified (increasing or reducing). In another words, the algorithm switches the modulation with the goal to improve the spectrum efficiency, thus, satisfying given QoT requirements. The number of, $k$, lightpath among source and destination is not fixed, instead it will also vary dynamically from one connection request to another. If a algorithm does not find a appropriate modulation for a determinate lightpath, the connection requested will be blocked and then the process is finalized.

The following considerations have been realized: we denote $n=1,2,3, \ldots, \mathbf{N}$ (with $\mathbf{N}$ the set of natural numbers), number of connections requests. For every connection request, $k$-Shortest paths can be selected between source and destination, with $k=1,2,3, \ldots, \boldsymbol{K}, D_{k}$ is the distance between source and destination for a determinate lightpath, with $D_{k}<D_{k+1}$. The maximum transmission distance reaches by each calculated modulations is recorded in $D_{M O D i}(\mathrm{i}=2,4,8,16,32,64$ corresponding to 64QAM, 32QAM, 16QAM, 8PSK, QPSK, and BPSK modulations, respectively). Variable, $E_{k}$, is the energy consumption of a determinate lightpath. A determined modulation for a considered lightpath is selected if $D_{k}$ is less than or equal to $D_{M O D i}$ or $D_{k}$ is more than $D_{M O D i-1}$ and less than or equal to $D_{M O D i}(i-1$ will correspond to the modulation just before a determined modulation $i$ ). Toward this end, a new SEC-RSA algorithm is introduced.

1) Receive, $n$, connections requests and randomly choose two nodes, one as source and the other as destination;

2) Establish the lightpaths from source to destination for every connections requests;

3) For $n=1$ execute Yen's algorithm and select the shortest path $D_{1}$. The shortest path must have less distance and less hops;

4) Compute the maximum distance of transmission reached for each modulations and record in $D_{M O D i}$;

5) Compare $D_{k}$ to $D_{M O D i}$ in order to select the ideal modulation, if any modulation is not found, the value of bit rate is increased. If SEC-RSA still does not find an ideal modulation, the connection is blocked and, then, finalize the process; otherwise, the process continues;

6) Compute the energy consumption for the connections requested;

7) Record the order of modulation selected and the computed energy consumption;

8) Run again the above iterations from item 2 to 7 except iteration 4 for $D_{2}, D_{3}, D_{4}, \ldots, D_{\mathbf{K}}$;

9) Compare and select the lightpath which satisfies QoS and QoT criterion, then, record the order of, $k$, and the lowest value of energy consumption $E_{k}$;

10) Check if still there are more connections requests; 
11) If it is affirmative, repeat all the process above from item 3 otherwise, end the process.

The output of the SEC-RSA algorithm will be the lightpath with shortest path, less hops and lowest energy consumption.

\section{RESUlTS AND DISCUSSION}

In order to evaluate the effectiveness of SEC-RSA algorithm and its impact on the energy consumption, an extensive simulations using USA nation wide network topology was conducted, which is illustrated in Fig.2 [4].

To create a dynamic scenario, the connections requests arrive one by one following a Poisson process, with $\lambda$ as a average arrival rate. The holding time is negative exponentially distributed.

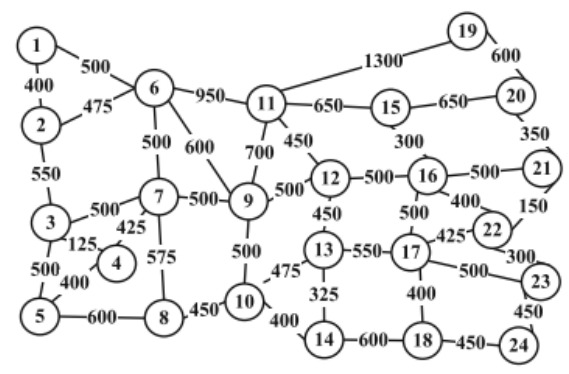

Fig. 2. US nation wide network

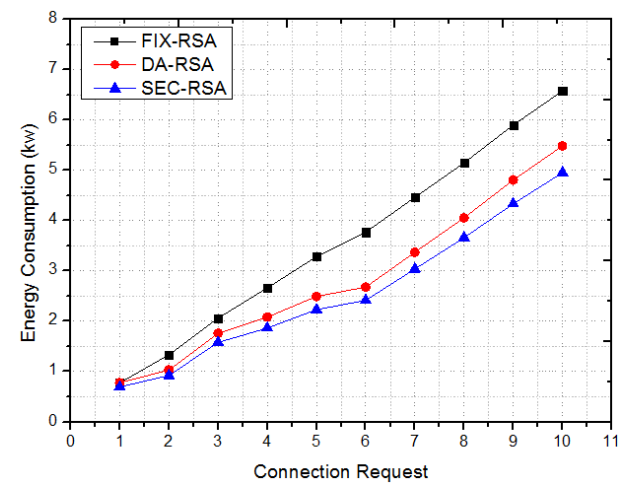

Fig. 3. Energy consumption versus Connections Requests for different Algorithms

The results have been compared with the traditional FIX-RSA (where fix BSK modulation is employed) and DA-RSA algorithm based on distance adaptive modulation [4]. For this first case there is no connection block. It means that, whenever the distance of a determinate lightpath is greater than that of the QPSk, the algorithm will consider the distance of the BPSK modulation and it will be chosen. Every algorithm will operate as the following scenarios for an incoming request:

- FIX-RSA will only employ fix BPSK modulation for incoming all connections.
- DA-RSA adopts the format-flexible transponder and chooses different modulation formats according to the physical distance between two adjacent nodes with the objective to improve the spectrum efficiency.

- SEC-RSA firstly executes Yen's Algorithm in order to select k-shortest path. Allocate the ideal modulation for every k selected shortest path. And finally selected the one with lowest energy consumption.

Fig. 3 represents the energy consumption in function of the connections requests. At least ten connections requests have to be routed in the EON. The simulations results clearly show that as the number of connection requests increases, the energy consumption also increases. Furthermore, SECRSA presents the lowest energy consumption compared with the others algorithms, because the SEC-RSA algorithm selects the best modulation format adaptively for the request according to the physical distance of the traversed link. Additionally, the algorithm will adaptively varies the, $k$, value according to the connections requests.

With the goal to highlight the influence of the modulation level, Fig.4 illustrates the total energy consumption versus connections requests for different modulation, excluding FEC effects. Every modulation formats route all the 1000 connections requests. The energy consumption of the optical network increases as the connections requests also increases. By increasing the modulation format, the power consumption decreases. BPSK modulation reveals the highest power consumption following by QPSK. On the other side, 64QAM shows the lowest energy consumption, once higher modulation level occupies less frequency slots, than the lower one, which helps to save spectrum resources, due to the flexible selection of the modulation format. Furthermore, the energy consumption at transmitter, in passive devices, as well as overall network energy consumption are reduced.

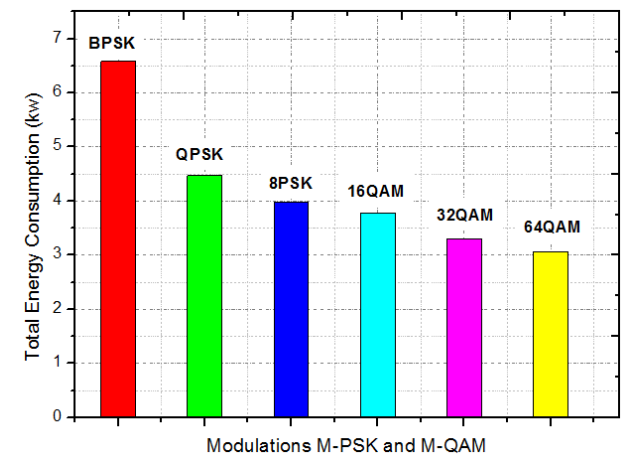

Fig. 4. Energy consumption for M-QAM and M-PSK modulations without FEC code.

Now, the possibility to have connections blocking in the EON are included in the simulations. There are connections blocks when if no proper modulation format is selected in order words, if the lightpath distance is greater than the MDT of BPSK. The blocking probability can be defined as the ratio between the number of request connection failed to the 
total connections number that arrives in the EON. Again, 1000 connections requests arrive in the EON. Fig.5 illustrates the total energy consumption for the three algorithms. Once again, SEC-RSA algorithm presents better performance in therms of energy consumption.

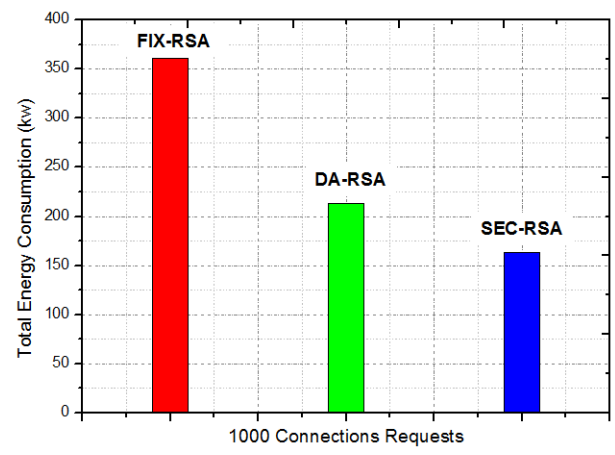

Fig. 5. Total Energy Consumption for 1000 connections requests for the three algorithms.

Table 1 summarizes the blocking probability and the total energy consumption $(\mathrm{kW})$ for every algorithms. This Table points out that SEC-RSA algorithm presents the lowest energy consumption when compared with FIX-RSA and DARSA. FIX-RSA depicts high blocking probability however, both SEC-RSA and DA-RSA have the same blocking probability. SEC-RSA and DA-RSA are the same blocking probability since the criteria to block incoming request are similar.In SEC-RSA algorithm the blocking probability can be reduced by increasing the transmission rate. The increment of the transmission rate drive to reach more nodes when connections requests arrive to the EON. However, the energy consumption of the EON will increase in that case. Thus, it means that there are values of transmission rates in what SEC-RSA will perform better than DA-RSA algorithms in therms of energy consumption and blocking probability. In future work we will look for the threshold based on transmission rate for our SEC-RSA algorithm.

TABLE I

TABLE OF BLOCKING PROBABILITY AND ENERGY CONSUMPTION

\begin{tabular}{|c|c|c|}
\hline Algorithms & Blocking Probability & Energy Consumption \\
\hline FIX - RSA & 0,578 & 361,22 \\
\hline DA - RSA & 0,378 & 213,54 \\
\hline SEC - RSA & 0,378 & 169,56 \\
\hline
\end{tabular}

Finally, the performance of SEC-RSA in therms of energy consumption is compared considering that all connections requests are successfully attended for one side and there are blocking connections on the order side. Fig.6 spotlights that energy consumption for SEC-RSA with blocking connections requests are lower than the other case with blocking probability equal 0.4 . It means that, for 10 connections requests, 6 connections were attended, while all the 10 connections requests are attended if any blocking connection in the EON is considered.

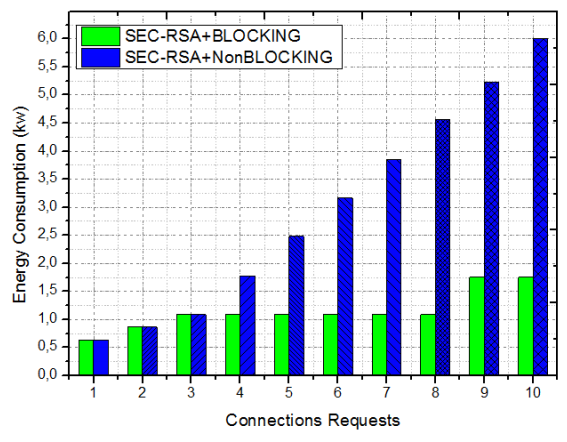

Fig. 6. Energy consumption Comparison for blocking and nonBlocking connection request

Therefore, the proposed SEC-RSA is good way to reduce energy consumption in EON at the same time ensures QoT requirements. The weakness of this proposal algorithm is the average run time, which is higher than DA-RSA and FIX-RSA algorithms. The reason is that the time for the modulation format selection in our scheme, including the time to look for the $k$-shortest path, will need various iterations for each connection request, which is longer than the others algorithms. However, SEC-RSA is preferred since it consumes less energy than DA-RSA and FIX-RSA.

\section{CONCLUSION}

Through a novel SEC-RSA algorithm, energy consumption can be reduced in EONs. The results also demonstrated that as the order of modulations formats increases, the energy consumption of the EON decreases. Energy consumption decreases when connections blocks in the EONs are considered. In future work, this novel algorithm will be applied in order to compute energy efficiency in EONs.

\section{ACKNOWLEDGMENT}

This work was supported by FAPEMIG, CNPQ and CAPES

\section{REFERENCES}

[1] G. Koutitas and P. Demestichas, "A review of energy efficiency in Telecommunication Networks", Telfor Journal, Vol. 2, No. 1, 2010.

[2] D. Maheswaran and K. K. J. Kailas, "Energy efficiency in electrical systems", IEEE International Conference on Power Electronics, Drives and Energy Systems, Bengaluru, India, Dec. 16-19, 2012

[3] T. Rodney, "Green optical communicationsPart I: Energy limitations in transport", IEEE Journal of selected topics in quantum electronics, v. 17, n. 2, p. 245-260, 2011.

[4] Z. Jijun et al, "Distance-adaptive routing and spectrum assignment in OFDM-based flexible transparent optical networks", Photonic Network Communications 27.3, p. 119-127, 2014.

[5] Ricciardi et al., "An energy-aware dynamic RWA framework for nextgeneration wavelength-routed networks", Computer Networks, v. 56, n. 10 , p. 2420-2442, 2012.

[6] M. Jinno, H. Takara, B. Kozicki, Y. Tsukishima, Y. Sone, and S. Matsuoka,"Spectrum-efficient and scalable elastic optical path network: Architecture, benefits, and enabling technologies, IEEE Commun. Mag., vol. 47,no. 11, pp. 6673, Nov. 2009

[7] K. Christodoulopoulos, I. Tomkos, and E. Varvarigos, "Elastic bandwidth allocation in flexible OFDM-based optical networks, IEEE J. Lightw. Technol., vol. 29, no. 9, pp. 13541366, May 2011.

[8] F. Durand and A. Taufik, "Energy efficiency analysis in adaptive FECbased lightpath elastic optical networks", Journal of Circuits, Systems and Computers, v. 24, n. 09, p. 1550133, 2015. 